\title{
A companion to AB Pic at the planet/brown dwarf boundary ${ }^{\star}$
}

\author{
G. Chauvin ${ }^{1}$, A.-M. Lagrange ${ }^{2}$, B. Zuckerman ${ }^{3}$, C. Dumas ${ }^{1}$, D. Mouillet ${ }^{4}$, I. Song ${ }^{3}$, J.-L. Beuzit ${ }^{2}$, \\ P. Lowrance ${ }^{5}$, and M. S. Bessell ${ }^{6}$
}

1 European Southern Observatory, Casilla 19001, Santiago 19, Chile e-mail: gchauvin@eso.org

${ }^{2}$ Laboratoire d'Astrophysique, Observatoire de Grenoble, 414 rue de la piscine, Saint-Martin-d'Hères, France

3 Department of Physics \& Astronomy and Center for Astrobiology, University of California, Los Angeles, Box 951562, CA 90095, USA

${ }^{4}$ Laboratoire d'Astrophysique, Observatoire Midi-Pyrénées, Tarbes, France

5 Spitzer Science Center, Infrared Processing and Analysis Center, MS 220-6, Pasadena, CA 91125, USA

${ }^{6}$ Research School of astronomy and Astrophysics Institue of Advance Studies, Australian National University, Cotter Road, Weston Creek, Canberra, ACT 2611, Australia

Received 24 March 2005 / Accepted 19 April 2005

\begin{abstract}
We report deep imaging observations of the young, nearby star AB Pic, a member of the large Tucana-Horologium association. We have detected a faint, red source 5.5" South of the star with JHK colors compatible with that of a young substellar L dwarf. Follow-up observations at two additional epochs confirm, with a confidence level of $4.7 \sigma$, that the faint red object is a companion to $\mathrm{AB}$ Pic rather than it being a stationary background object. A low resolution $K$-band spectrum indicates an early-L spectral type for the companion. Finally, evolutionary model predictions based on the $J H K$ photometry of AB Pic b indicate a mass of 13 to $14 M_{\text {Jup }}$ if its age is $\sim 30$ Myr. Is AB Pic b a massive planet or a minimum mass brown dwarf?
\end{abstract}

\section{Introduction}

In October 2000, we began a deep imaging survey of stars in young, nearby southern associations to search for companion brown dwarfs and giant planets. First, we used the ADONIS/SHARPII adaptive optics (AO) instrument at the ESO/3.6 m telescope (Chauvin et al. 2003). Since November 2002 we have pursued the survey with the VLT/NACO instrument. We mainly focussed our search on the Tucana-Horologium (Torres et al. 2000; Zuckerman \& Webb 2000) and TW Hydrae (Kastner et al. 1997) associations, as well as the $\beta$ Pictoris (Zuckerman et al. 2001) and AB Doradus (Zuckerman et al. 2004) co-moving groups, to explore the circumstellar environment within semimajor axes between tens and hundreds of AU with detection limits down to a few Jupiter masses.

This strategy resulted in the astrometric and spectroscopic confirmation of a brown dwarf companion to GSC 08047-00232 (Chauvin et al. 2005; Neuhäuser \& Guenther 2004) and detection of a giant planet companion candidate to the young brown dwarf 2M1207 (Chauvin et al. 2004, 2005, accepted).

In March 2003, we observed the young star AB Pic (HIP 30034, K2V, $V=9.16, d=47.3_{-17}^{+1.8} \mathrm{pc}$ ), identified by Song et al. (2003) as a member of the large Tucana-Horologium

^ Based on ESO observing programs 70.C-0677(B), 072.C-0644(B) and 073.C-0469(B) at the VLT. association of estimated age $\sim 30 \mathrm{Myr}$, according to a comparison of $V-K$ versus $M_{K}$ with evolutionary tracks. At 5.5" South from AB Pic, we detected (Fig. 1) a faint and red object with a near-IR color compatible with that observed for cool L dwarfs (Leggett et al. 2002). We re-observed both objects in March and September 2004 in order to determine if they shared common proper motions. In December 2004, acquisition of a near-IR spectrum enabled us to determine the spectral type of AB Pic b and to confirm its substellar status.

\section{Observations}

AB Pic A and b were imaged with NACO using classical imaging and coronagraphy (Table 1 and Fig. 2). The JHK photometry of AB Pic A is known from the 2MASS All-Sky Catalog of Point Sources (Cutri et al. 2003). On 17 March 2003, we measured the JHK contrasts of AB Pic A and b, using the deconvolution algorithm of Véran \& Rigaut (1998), so as to determine the photometry of AB Pic b (see Table 2). The transformation between the $K_{\mathrm{s}}$ filter of NACO and the $K$ filter used by CTIO-2MASS was found to be smaller than $0.03 \mathrm{mag}$. On 5 March 2004 and 25 September 2004, follow-up observations of $\mathrm{AB}$ Pic $\mathrm{A}$ and $\mathrm{b}$ were acquired in the $H$ band with the S13 camera to provide the best compromise between high angular resolution and AO correction. To calibrate the platescale and the detector orientation, we observed at each epoch the astrometric field of $\theta$ Ori $1 \mathrm{C}$. The orientations of true north of the 
Table 1. Observing Log.

\begin{tabular}{|c|c|c|c|c|c|c|c|c|c|}
\hline Name & UT date & Tot. exp. time & Filter & Camera & Mode & $\begin{array}{l}\text { Strehl } \\
(\%)\end{array}$ & $\begin{array}{l}\text { Seeing } \\
(\operatorname{arcsec})\end{array}$ & Airmass & Remarks \\
\hline \multicolumn{10}{|c|}{ Classical imaging and coronagraphy } \\
\hline \multirow[t]{3}{*}{ AB Pic A } & $17 / 03 / 2003$ & $60 \times 2 s$ & NB1.24 & $\mathrm{S} 13$ & classical & 12 & 0.75 & 1.32 & science \\
\hline & $17 / 03 / 2003$ & $120 \times 0.5 \mathrm{~s}$ & NB1.75 & $\mathrm{S} 13$ & classical & 25 & 0.80 & 1.22 & science \\
\hline & $17 / 03 / 2003$ & $100 \times 0.35 \mathrm{~s}$ & NB2.17 & S27 & classical & 32 & 0.80 & 1.31 & science \\
\hline \multirow[t]{3}{*}{ AB Pic b } & $17 / 03 / 2003$ & $18 \times 30 \mathrm{~s}$ & $J$ & $\mathrm{~S} 13$ & coronagraphy & 12 & 0.75 & 1.27 & science \\
\hline & $17 / 03 / 2003$ & $5 \times 30 \mathrm{~s}$ & $H$ & $\mathrm{~S} 13$ & coronagraphy & 25 & 0.80 & 1.28 & science \\
\hline & $17 / 03 / 2003$ & $3 \times 20 s$ & $K_{\mathrm{s}}$ & $\mathrm{S} 27$ & coronagraphy & 32 & 0.80 & 1.30 & science \\
\hline$\theta$ Ori $1 \mathrm{C}$ & $16 / 03 / 2003$ & $12 \times 10 \mathrm{~s}$ & NB1.75 & S13 & coronagraphy & 37 & 1.00 & 1.12 & astrometric std \\
\hline AB Pic A & 05/03/2004 & $10 \times 1 \mathrm{~s}$ & NB1.75 & $\mathrm{S} 13$ & classical & 20 & 1.00 & 1.20 & science \\
\hline AB Pic b & 05/03/2004 & $4 \times 30 \mathrm{~s}$ & $H$ & $\mathrm{~S} 13$ & coronagraphy & 20 & 1.10 & 1.20 & science \\
\hline$\theta$ Ori $1 \mathrm{C}$ & $05 / 03 / 2004$ & $10 \times 12 \mathrm{~s}$ & $H$ & $\mathrm{~S} 13$ & coronagraphy & 38 & 1.10 & 1.15 & astrometric std \\
\hline AB Pic A & $25 / 09 / 2004$ & $2 \times 5 s$ & $H+\mathrm{ND}$ & $\mathrm{S} 13$ & classical & 27 & 0.74 & 1.28 & science \\
\hline AB Pic b & $25 / 09 / 2004$ & $3 \times 20 \mathrm{~s}$ & $H$ & $\mathrm{~S} 13$ & coronagraphy & 27 & 0.75 & 1.28 & science \\
\hline$\theta$ Ori $1 \mathrm{C}$ & $25 / 09 / 2004$ & $4 \times 0.8 \mathrm{~s}$ & $H$ & $\mathrm{~S} 13$ & coronagraphy & 24 & 0.85 & 1.15 & astrometric std \\
\hline \multicolumn{10}{|c|}{ Spectroscopy } \\
\hline AB Pic b & $03 / 12 / 2004$ & $16 \times 300 \mathrm{~s}$ & SHK & S54 & $R_{\lambda}=550$ & na & 0.87 & 1.26 & science \\
\hline HIP 33632 & 03/12/2004 & $10 \times 5 \mathrm{~s}$ & SHK & S54 & $R_{\lambda}=550$ & na & 0.70 & 1.08 & telluric std \\
\hline
\end{tabular}

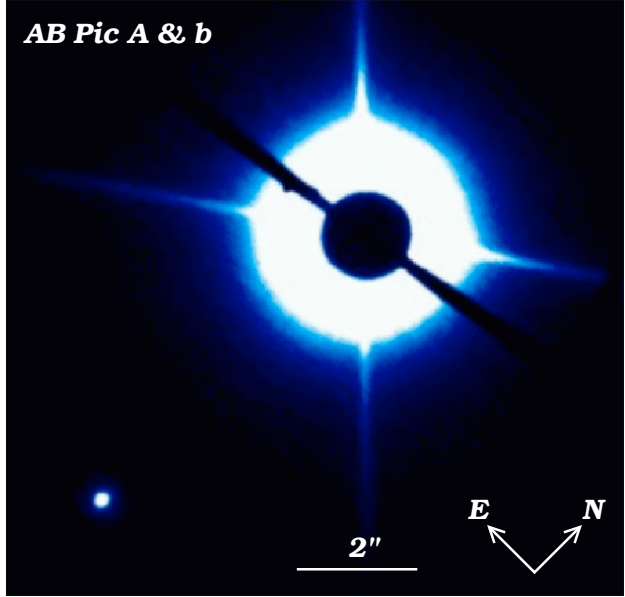

Fig. 1. $K_{\mathrm{s}}$-band coronagraphic image of $\mathrm{AB}$ Pic $\mathrm{A}$ and b acquired on 17 March 2003 with an occulting mask of diameter 1.4".

S13 camera were found on 16 March 2003, 5 March 2004 and 25 September 2004 respectively at $-0.05^{\circ}, 0.04^{\circ}, 0.20^{\circ}$ east of the vertical with an uncertainty of $0.10^{\circ}$. The pixel scale was found to be relatively stable in time with values of $13.21 \pm$ 0.11 mas, $13.24 \pm 0.05$ mas and $13.23 \pm 0.09$ mas.

The NACO spectroscopic observations of $\mathrm{AB}$ Pic $\mathrm{b}$ were obtained on 3 December 2004, using the low resolution $\left(R_{\lambda}=\right.$ 550) grism with the 86 mas slit, the S54 camera (54 mas/pixel) and the SHK filter covering the entire spectral range between 1.39 and $2.52 \mu \mathrm{m}$. The telluric standard star HIP 33632 (B6V) was also observed. After substracting the sky and dividing by a flat field using eclipse (Devillard 1997), the spectra of AB Pic $b$ and HIP 33632 were extracted and calibrated in wavelength with IRAF/DOSLIT. To calibrate the relative throughput
Table 2. Photometry of AB Pic A and b.

\begin{tabular}{llll}
\hline \hline Component & $\begin{array}{l}J \\
(\mathrm{mag})\end{array}$ & $\begin{array}{l}H \\
(\mathrm{mag})\end{array}$ & $\begin{array}{l}K \\
(\mathrm{mag})\end{array}$ \\
\hline $\mathrm{AB} \mathrm{Pic}^{a}$ & $7.58+-0.03$ & $7.09+-0.03$ & $6.98+-0.03$ \\
$\mathrm{AB} \mathrm{Pic} \mathrm{b}^{b}$ & $16.18+-0.10$ & $14.69+-0.10$ & $14.14+-0.08$ \\
\hline
\end{tabular}

${ }^{a}$ From the 2MASS All-Sky Catalog of Point Sources (Cutri et al. 2003).

${ }^{b}$ From ${ }^{a}$ and NACO measurements presented in this work.

of the atmosphere and the instrument, we divided the extracted spectrum of AB Pic b by the spectrum of HIP 33632. To restore the continuum shape, we then multiplied by a composite spectrum of a B6IV star taken from a library of stellar spectra (Pickles 1998).

\section{Companionship confirmation}

To verify that $\mathrm{AB}$ Pic $\mathrm{A}$ and $\mathrm{b}$ were comoving together in the sky and thus physically bound, their relative positions were determined on 17 March 2003, 5 March 2004 and 25 September 2004 (see Table 3). We then took into account the proper motion of AB Pic A from the Tycho catalog Høg et al. (2000): $\mu_{\alpha}=15.9 \pm 1.2 \mathrm{mas} / \mathrm{yr}$ and $\mu_{\delta}=46.2 \pm 1.2 \mathrm{mas} / \mathrm{yr}$, its expected parallactic motion and the detector calibrations at each epoch (platescale and detector orientation, see Sect. 2). The expected variations in separation and position angle in the case of a bound companion and of a background stationary object are shown in Fig. 2. The maximal orbital motion of AB Pic b from March 2003 to September 2004 is $<12$ mas. In the case of a stationary background object, important variations 

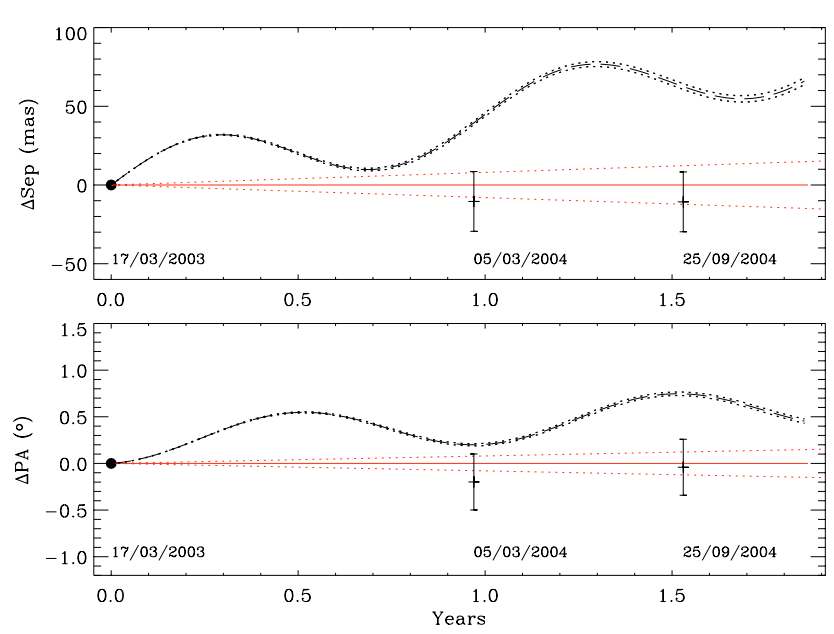

Fig. 2. Top: differences of separation between $\mathrm{AB}$ Pic A and b on 17 March 2003, 5 March 2004, and 25 September 2004. Bottom: differences of position angle for AB Pic A and b. The expected evolutions with their uncertainties for bound (solid line) and stationary background (dashed line) objects are indicated.

Table 3. Astrometric measurements of $\mathrm{AB} P \mathrm{Pic} A$ and $\mathrm{b}$ and confidence level estimation that $\mathrm{AB}$ Pic $\mathrm{b}$ is not a stationary background object.

\begin{tabular}{llll}
\hline \hline UT date & $\begin{array}{l}\text { Separation } \\
(\mathrm{mas})\end{array}$ & $\begin{array}{l}\text { Position angle } \\
\left({ }^{\circ}\right)\end{array}$ & $\begin{array}{l}\text { Confidence } \\
\text { level }\end{array}$ \\
\hline $17 / 03 / 2003$ & $5460 \pm 14$ & $175.33 \pm 0.18$ & - \\
$05 / 03 / 2004$ & $5450 \pm 16$ & $175.13 \pm 0.21$ & $3.0 \sigma$ \\
$25 / 09 / 2004$ & $5450 \pm 14$ & $175.30 \pm 0.20$ & $4.7 \sigma$ \\
\hline
\end{tabular}

are expected in both separation and position angle. Between March 2003 and September 2004, differences of separation and position angle show that AB Pic b is not a stationary background object, at the $4.7 \sigma$ confidence level.

\section{Spectral type determination}

Based on the near-IR photometry presented in Table 2, we can derive the near-IR colors of AB Pic b: $(J-K)=2.04,(J-H)=$ 1.49 and $(H-K)=0.55$. These values are consistent with that observed by Knapp et al. (2004) for late-type L dwarfs.

From spectroscopy, we decided to consider only the $K$-band portion of the AB Pic b spectrum which is less subject than the $H$-band to chromatical effects, mainly due to differential slit centering and/or differential AO corrections between the science source and the telluric standard (see Goto et al. 2002; Chauvin et al. 2004). Based on the template spectra for late-M, L and T dwarfs of Leggett et al. (2001) and Geballe et al. (2002), we used a minimum $\chi^{2}$ adjustment to find the best template spectra matching the broad water-band absorptions of the $\mathrm{AB}$ Pic $\mathrm{b}$ spectrum. This allowed us to derive a spectral type $\mathrm{L}_{-1}^{+2}$ for $\mathrm{AB}$ Pic b. The best adjustment is obtained with the L1 dwarf 2MASS J0345+2540 (see Fig. 3). In addition, various water band indices confirm this spectral type estimation. The water band index $Q$ of Wilking et al. (1999) is equal to 0.44 for $\mathrm{AB} P$ ic $\mathrm{b}$ which confirms a spectral type later than M 9. The $K 1$ and $K 2$ indices of Reid et al. (2001)

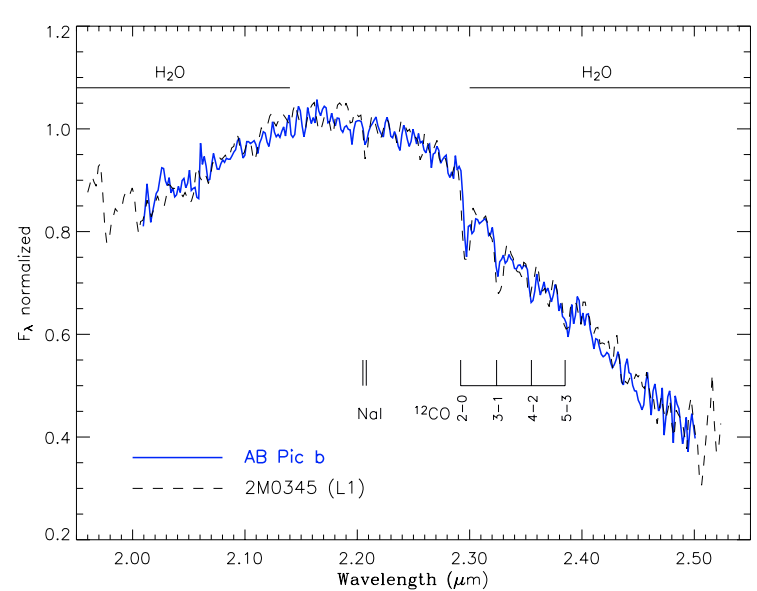

Fig. 3. $K$-band spectrum of AB Pic b acquired on 3 December 2004 with the low resolution $\left(R_{\lambda}=550\right)$ grism of NACO, the 86 mas slit and the 554 camera (54 mas/pixel). The best $\chi^{2}$ adjustment is found with the L1 dwarf 2MASSJ0345+2540 (Geballe et al. 2002).

are respectively equal to 0.23 and -0.03 . The $\mathrm{H}_{2} \mathrm{O} 2.0 \mu \mathrm{m}$ index of Geballe et al. (2002) is equal to 1.15. They all confirm a spectral type L0 to L3 for AB Pic b. The presence of absorption lines of NaI $(2.205$ and $2.209 \mu \mathrm{m})$ and the ${ }^{12} \mathrm{CO}$ transitions 2-0 (2.295 $\mu \mathrm{m}), 3-1(2.324 \mu \mathrm{m}), 4-2(2.354 \mu \mathrm{m})$ and 5-3 $(2.385 \mu \mathrm{m})$ also support the fact that AB Pic b is an early-L dwarf.

\section{Temperature and mass determination}

As an $\mathrm{L}_{-1}^{+2}$ dwarf companion to a $30 \mathrm{Myr}$ old star, AB Pic b is unambiguously a substellar object. To investigate its physical properties (mass, temperature and luminosity), evolutionary model predictions can be compared to the absolute photometry of AB Pic b. However, as described by Baraffe et al. (2002), model predictions must be considered carefully as they are still uncertain at early phases of evolution $(\leq 100 \mathrm{Myr}$; see also Mohanty et al. 2004; and Close et al. 2005).

We then considered the most commonly used models of Burrows et al. (1997), Chabrier et al. (2000) and Baraffe et al. (2002) to describe the atmospheric properties of young brown dwarfs and giant planets. Table 4 displays predictions of DUSTY models of Baraffe, of Burrows et al. (1997) and B. Macintosh \& M. Marley (personal communication). As may be seen, for a 30 Myr old object, the two calculations agree rather well, with one a bit redder in $J-K$ than AB Pic b and the other a bit bluer.

\section{Gravitational collapse or core accretion?}

With a model-derived mass of about $13 M_{\text {Jup }}$, one can speculate whether AB Pic b is a giant planet or a brown dwarf. The International Astronomical Union has recently adopted a definition to differentiate planetary and brown dwarf companions. The latter are objects with masses above the minimum mass for deuterium burning (13.6 $M_{\text {Jup }}$ ). Based on this criterion, as one may see from Table 4 , it is going to be very difficult to decide between very high mass planet or a very low mass brown dwarf, 
Table 4. Absolute magnitudes of AB Pic b compared to evolutionary model predictions (Burrows et al. 1997; Baraffe et al. 2002; B. Macintosh \& T. Barman, private communication).

\begin{tabular}{lllllll}
\hline \hline Model & $\begin{array}{l}\text { Age } \\
(\mathrm{Myr})\end{array}$ & $\begin{array}{l}\text { Mass } \\
\left(M_{\mathrm{Jup}}\right)\end{array}$ & $\begin{array}{l}T_{\text {eff }} \\
(\mathrm{K})\end{array}$ & $\begin{array}{l}M_{J} \\
(\mathrm{mag})\end{array}$ & $\begin{array}{l}M_{H} \\
(\mathrm{mag})\end{array}$ & $\begin{array}{l}M_{K} \\
(\mathrm{mag})\end{array}$ \\
\hline AB Pic b & 30 & & & 12.80 & 11.37 & 10.76 \\
\hline Burrows $^{*}$ & 30.4 & 13 & 1513 & 12.8 & 11.9 & 11.2 \\
& 30.4 & 14 & 1856 & 11.5 & 10.7 & 10.1 \\
\hline DUSTY $^{*}$ & 30 & 13 & 1594 & 14.0 & 12.2 & 11.0 \\
& 30 & 14 & 1764 & 12.8 & 11.5 & 10.5 \\
& 20 & 13 & 1672 & 13.2 & 11.7 & 10.7 \\
& 40 & 14 & 1701 & 13.3 & 11.8 & 10.7 \\
\hline
\end{tabular}

* The gravity factor $\log (g)$ is respectively equal to 4.0 and 4.1 for the Burrows and DUSTY models.

given uncertainties in evolutionary models and in the age of AB Pic b. A second criterion, defended by an important fraction of the community, would be to differentiate planet from brown dwarf according to their origins of formation. But, more than discussing the definition of AB Pic $b$, everyone should agree that a more meaningful question is here to know whether this object has formed by gravitational collapse, that is, in a onestep process, or by a two-step process that begins with core accretion.

An answer is suggested by early results from imaging surveys for young planets in combination with data from the precision radial velocity technique (PRV). In the very close stellar environment, closer than $\sim 4$ AU, PRV results indicate a mass spectrum that increases toward lower masses, from $10 M_{\text {Jup }}$ to less than $1 M_{\text {Jup }}$ (see Jorissen et al. 2001). In contrast, our VLT survey of $\sim 50$ stars, which is sensitive to masses down to about $2 M_{\text {Jup }}$ at physical separations wider than $\sim 80 \mathrm{AU}$, has revealed only the possible planetary mass companion $\mathrm{AB}$ Pic $\mathrm{b}$ ( $\sim 13 M_{\text {Jup }}$ at $\left.\sim 260 \mathrm{AU}\right)$. (The case of $2 \mathrm{M} 1207 \mathrm{~b}$ is not considered in the discussion as the primary is a young brown dwarf). In addition, no other sensitive near-infrared imaging survey of young stars - with VLT/NACO (Masciadri et al. 2005), with the Keck AO system (Macintosh et al., in preparation) and with HST (Song et al., in preparation) - has yet reported any objects of planetary mass. Thus, this apparent absence of companions of a few Jupiter masses, suggests that wide companions like AB Pic b have formed in situ by gravitational collapse. In addition, for separations as large as $\sim 260 \mathrm{AU}$, formation by core accretion of planetesimals seems very unlikely because of inappropriate timescales to form planetesimals at such distances (Augereau et al. 2001). Gravitational instabilities within a protoplanetary disk (Papaloizou \& Terquem 2001; Rafikov 2005) or mechanisms proposed for brown dwarfs formation (Kroupa \& Bouvier 2003) appear to be more probable scenarii to explain the origin of wide companions such as AB Pic b.

Interestingly, gravitational collapse mechanisms, which are relatively insensitive to metallicity, may also be true for very massive PRV planets with small semi-major axes ( $<4 \mathrm{AU})$. Inspection of stellar metallicity vs planet mass data given in Santos et al. (2004) and Fischer \& Valenti (2005) indicates that the well-known correlation between high stellar metallicity and the existence of planets may not be present for stars with the highest mass planets (>7 $M_{\text {Jup }}$; see also Rice et al. 2003). That is, these relatively few highest mass PRV planets may have formed by gravitational collapse, while the lower mass PRV planets formed mostly or entirely via core accretion with subsequent gravitational infall. As the number of systems, detected by PRV and direct imaging, continues to grow, it should be possible to confirm or deny these tentative correlations.

Acknowledgements. We would like to thank the staff of the ESO, Gilles Chabrier, Isabelle Baraffe and France Allard for providing the latest update of their evolutionary models, Sandy Leggett and Tom Geballe who kindly sent us their near-infrared template spectra, and finally Sandy Leggett again for her remarks on the letter.

\section{References}

Augereau, J.-C., Nelson, R. P., Lagrange, A.-M., Papaloizou, J. C. B., \& Mouillet, D. 2001, A\&A, 370, 447

Baraffe, I., Chabrier, G., Allard, F., \& Hauschildt, P. H. 2002, A\&A, 382,563

Burrows, A., Marley, M., Hubbard, W. B., et al. 1997, AJ, 491, 856

Chabrier, G., Baraffe, I., Allard, F., \& Hauschildt, P. H. 2000, ApJ, 542,464

Chauvin, G., Thomson, M., Dumas, C., et al. 2003, A\&A, 404, 157

Chauvin, G., Lagrange, A.-M., Dumas, C., et al. 2004, A\&A, 425, L29

Chauvin, G., Lagrange, A.-M., Lacombe, F., et al. 2005, A\&A, 430, 1027

Close, L. M., Lenzen, R., Guirado, J. C., et al. 2005, Nature, 433, 286 Cutri, R. M., Skrutskie, M. F., van Dyk, S., et al. 2003, 2MASS AllSky Catalog of Point Sources

Devillard, N. 1997, The messenger, 87

Fischer, D. A., \& Valenti, J. 2005, ApJ, 622, 1102

Geballe, T. R., Knapp, G. R., Leggett, S. K., et al. 2002, ApJ, 564, 466

Goto, M., Kobayashi, N., Terada, H., et al. 2002, ApJ, 567, L59

Høg, E., Fabricius, C., Makarov, V. V., et al. 2000, A\&A, 355, 27

Jorissen, A., Mayor, M., \& Udry, S. 2001, A\&A, 379, 992

Kastner, J. H., Zuckerman, B., Weintraub, D. A., \& Forveille, T. 1997, Science, 277, 67

Knapp, G. R., Leggett, S. K., Fan, X., et al. 2004, AJ, 127, 3553

Kroupa, P., \& Bouvier, J. 2003, MNRAS, 346, 369

Leggett, S. K., Allard, F., Geballe, T. R., Hauschildt, P. H., \& Schweitzer, A. 2001, ApJ, 548, 908

Leggett, S. K., Golimowski, D. A., Fan, X., et al. 2002, ApJ, 564, 452

Masciadri, E., Mundt, R., Henning, Th., \& Alvarez, C. 2005, ApJ [arXiv: astro-ph/0502376]

Mohanty, S., Jayawardhana, R., \& Basri, G. 2004, ApJ, 609, 885

Neuhäuser, R., \& Guenther, E. W. 2004, A\&A, 420, 647

Papaloizou, J. C. B., \& Terquem, C. 2001, MNRAS, 325, 221

Pickles, A. J. 1998, PASP, 110, 863

Rafikov, R. R. 2005, ApJ, 621, L69

Reid, I. N., Burgasser, A. J., Cruz, K. L., Kirkpatrick, J. D., \& Gizis, J. E. 2001, AJ, 121, 1710

Rice, W., Armitage, P., Bonnell, I., et al. 2003, MNRAS, 346, L36

Santos, N. C., Israelian, G., \& Mayor, M. 2004, A\&A, 415, 1153

Song, I., Zuckerman, B., \& Bessel, M. S. 2003, ApJ, 599, 342

Torres, C. A. O., Da Silva, L., Quast, G. R., de la Reza, R., \& Jilinski, E. 2000, AJ, 120,1410

Véran, J. P., \& Rigaut, F. 1998, SPIE, 3353, 426

Wilking, B. A., Greene, T. P., \& Meyer, M. R. 1999, AJ, 117, 469

Zuckerman, B., \& Webb, R. A. 2000, ApJ, 535, 959

Zuckerman, B., Song, I., Bessel, M. S., \& Webb, R. A. 2001, ApJ, 562, L87

Zuckerman, B., Song, I., \& Bessel, M. S. 2004, ApJ, 613, L65 